\title{
Attention to Painful Stimulation Enhances $\gamma$-Band Activity and Synchronization in Human Sensorimotor Cortex
}

\author{
Michael Hauck, ${ }^{1}$ Jürgen Lorenz, ${ }^{2}$ and Andreas K. Engel ${ }^{1}$ \\ ${ }^{1}$ Department of Neurophysiology and Pathophysiology, University Medical Center Hamburg-Eppendorf, 20246 Hamburg, Germany, and ${ }^{2}$ Department of \\ Applied Natural Science, Laboratory of Human Biology and Physiology, Applied Science University, 21033 Hamburg, Germany
}

\begin{abstract}
A number of cortical regions are involved in processing pain-related information. The SI and SII somatosensory cortices process mainly sensory discriminative attributes but also play an important role in recognition and memory of painful events. Regions such as SII and the posterior insula appear to be the first stations that house processes by which attention profoundly shapes both behavioral responses and subjective pain experience. We investigated the influence of directed attention on pain-induced oscillations and synchronization processes using magnetoencephalogram in combination with an oddball paradigm in 20 healthy subjects. The subject's task was to count rare painful electrical stimuli applied to one finger, while ignoring frequent stimuli at a different finger. A high detection ratio was observed for all blocks and subjects. Early evoked oscillations in the $\delta$-band increased with higher stimulus intensity and directed attention, most prominently at contralateral sensorimotor sites. Furthermore, suppression and rebound of $\beta$ activity were observed after painful stimulation. Moreover, induced oscillatory activity in the high $\gamma$-band increased with directed attention, an effect being significantly stronger for high compared with low stimulus intensity. Coupling analysis performed for this high $\gamma$ response revealed stronger functional interactions between ipsilateral and contralateral sites during attention. We conclude that pain-induced high-frequency activity in sensorimotor areas may reflect an attentional augmentation of processing, leading to enhanced saliency of pain-related signals and thus to more efficient processing of this information by downstream cortical centers.
\end{abstract}

Key words: pain; attention; $\gamma$; oscillations; synchronization; SII; MEG

\section{Introduction}

The perception of pain is determined by the physical nature of the painful stimulus and the psychological context in which it occurs. A number of cortical regions are involved in processing painrelated information (Peyron et al., 2000). The primary (SI) and secondary (SII) somatosensory cortices process mainly sensorydiscriminative attributes, which provide information about the duration, strength, and location of pain, but also play an important role in recognition and memory of painful events (Schnitzler and Ploner, 2000). Regions such as SII and posterior insular cortices appear to be the first stations that house processes by which attention profoundly shapes both behavioral responses to pain and subjective pain experience (Lorenz and Garcia-Larrea, 2003). In clinical contexts, attention toward pain can aggravate chronic pain and the associated subjective experience (Aldrich et al., 2000; Eccleston et al., 2001). Furthermore, it can interfere with concurrent cognitive activities (Eccleston and Crombez, 1999). Therefore, studying the cerebral mechanisms of attentional modulation of pain processing can help to better understand the role

Received Jan. 23, 2007; revised June 28, 2007; accepted June 29, 2007.

This work was supported by Universitätsklinikum Hamburg-Eppendorf Junior Research Grant UKE FFM F-161-1 and European Union Grant NEST-2006-043457 "MindBridge." We thank Kriemhild Saha, Roger Zimmermann, and Gerhard Steinmetz for help during the experiments and Alexander Maye for helpful discussions.

Correspondence should be addressed to Dr. Michael Hauck, Department of Neurophysiology and Pathophysiology, University Medical Center Hamburg-Eppendorf, Martinistrasse 52, 20246 Hamburg, Germany. E-mail: hauck@uke.uni-hamburg.de.

DOI:10.1523/JNEUROSCI.2283-07.2007

Copyright $\odot 2007$ Society for Neuroscience $\quad$ 0270-6474/07/279270-08\$15.00/0 of psychological factors in pain and their associated therapeutic relevance.

Previous studies on the correlates of interactive stimulus and pain modulation effects in the electroencephalogram (EEG) or magnetoencephalogram (MEG) concentrated on slow eventrelated potentials after painful electrical or laser stimuli (for review, see Bromm and Lorenz, 1998; Lorenz and Garcia-Larrea, 2003). These studies substantiated the role of attention in pain processing and allowed the identification of separate and overlapping components sensitive to pain and task factors including EEG P3-like phenomena (Becker et al., 2000; Legrain et al., 2002; Dowman, 2004) (for review, see Lorenz and Garcia-Larrea, 2003). A promising approach to analyzing the internally driven dynamics of cortical pain processing is to evaluate oscillations that are induced by painful stimuli. In this context, particularly the $\gamma$-band $(>30 \mathrm{~Hz})$ has been the subject of numerous studies pursuing the hypothesis that binding of stimulus features into a coherent perception may occur in this frequency range (for review, see Gray and Singer, 1995; Singer, 1999; Tallon-Baudry and Bertrand, 1999; Engel and Singer, 2001) (data not shown). There is evidence suggesting causal links between the changes in oscillatory synchrony and the efficiency of stimulus processing by thalamocortical networks (Salinas and Sejnowski, 2001; Fries, 2005; Siegel et al., 2007; Womelsdorf et al., 2007). Top-down influences such as focused attention may act by modulating subthreshold oscillations in sensory assemblies and by enhancing the gain of oscillatory responses to stimuli that match stored contex- 


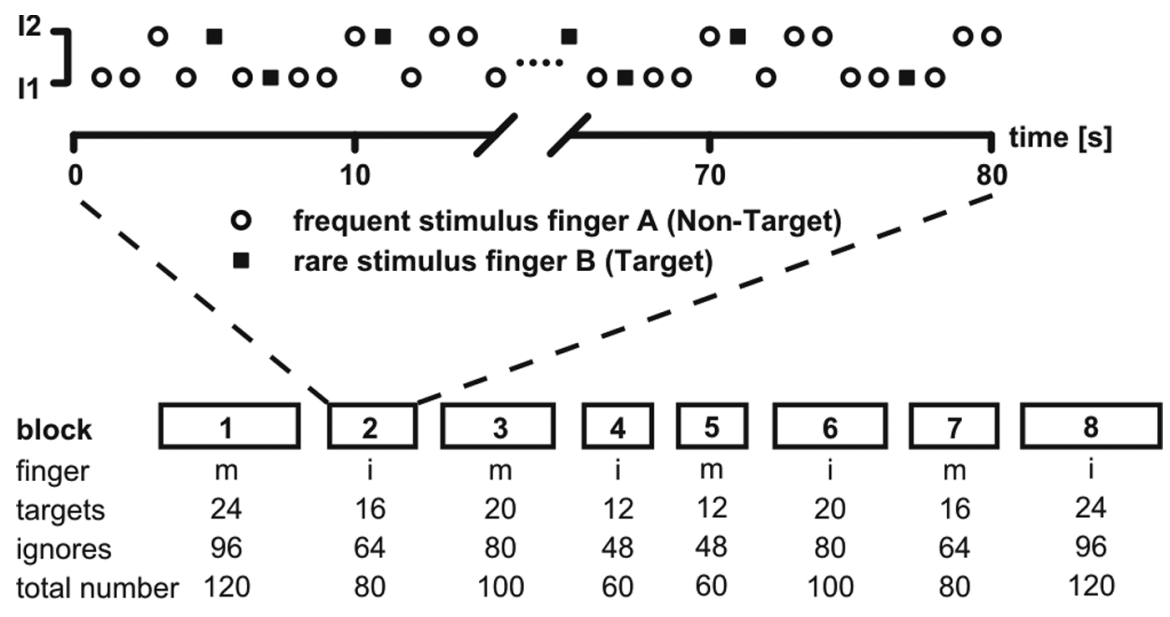

Figure 1. Experimental paradigm. The experimental setup consisted of eight blocks in total with variable block length. The first block (1) always started with the middle $(m)$ finger as target. The second block is shown in more detail in the top panel. Subjects had to count 16 target stimuli (squares) at the index (i) finger, which occurred randomly, while having to ignore 64 stimuli (circles) applied to the middle finger. Hence, this block consisted of 80 pain stimuli with two intensities, I1 (low, 50\%) and I2 (high, 50\%). Stimuli were delivered at a frequency of $1 \mathrm{~Hz}$ for all blocks.

tual information (Engel et al., 2001; Herrmann et al., 2004). Along the same lines, $\gamma$-band oscillations might also play an integral role in pain perception and processing (Chen and Herrmann, 2001; Croft et al., 2002; Ohara et al., 2006).

In the present study, we aimed at further characterizing painrelated fast oscillatory activity by manipulating pain intensity and task relevance independently. To this end, we used an oddball design using painful intracutaneous electrical stimuli applied at the index and middle fingers of healthy subjects. We recorded pain oscillations over both somatosensory cortices, studying their modulation by stimulus- and task-related factors.

\section{Materials and Methods}

Subjects. Before the start of the experiment, the protocol was approved by the local ethics review board. Twenty right-handed subjects (10 female, 10 male; age, $25.2 \pm 3.8$ years; height, $177.0 \pm 8.6 \mathrm{~cm}$; weight, $69.1 \pm 12.2$ $\mathrm{kg}$ ) participated in this study after written informed consent. Subjects were free to terminate the experiment at any time.

Pain stimuli. The intracutaneous pain model was used (Bromm and Meier, 1984) to induce pain. Briefly, a thin electrode was fed through a hole drilled into the epidermal skin at the tip of the index and middle finger in the nondominant hand, and thus electrical stimulus pulses (16 ms duration) could be delivered to the nearest proximity of nociceptors. The short electrical pulse mainly activates $A \delta$-fibers, whereas $\mathrm{C}$-fibers could not be activated because of their long chronaxie (Bromm and Meier, 1984). Before the experiment, individual pain thresholds were tested by determining the average intensity at which subjects reported a given stimulus as painful. This was done for each finger separately using five ascending and descending series of electric stimuli with successive intensity increments of $0.01 \mathrm{~mA}$. During the experiment, all stimuli were applied with an intensity 1.5 -fold of the pain threshold for the "low-pain" intensity and 2-fold of the threshold for the "high-pain" intensity.

Experimental protocol. Pain stimuli were applied in two intensities in pseudorandom order (high and low intensity, 50\% balanced) at a frequency of $1 \mathrm{~Hz}$. One of the two fingers received frequent (80\%) stimuli that the subjects had to ignore (i.e., nontargets), whereas the other finger received stimuli more rarely (20\%), which were defined as targets. An example for the stimulus sequence is illustrated in Figure 1. Subjects were instructed to silently count the targets and to report the number of perceived pain stimuli after each block, whereas the intensity of the stimuli was task irrelevant. There were eight blocks in total that varied in the overall number of stimuli between 60 and 120. In four blocks (blocks 2, 4, 6 , and 8 ), the target stimuli were applied at the index finger; whereas in the other four blocks (blocks 1, 3, 5, and 7), the target stimuli were delivered to the middle finger. Thus, the target sites for the stimuli were run in alternating order, and sequence was counterbalanced across participants. Targets appeared with a probability of $20 \%$ in each block. For each finger, a total of 72 target stimuli $(24,20,16$, or 12 per block, respectively) and 288 nontarget stimuli $(96,80,64$, or 48 per block) were presented resulting in 144 target and 576 nontarget stimuli for the entire experiment. Experimental blocks lasted between 60 and $120 \mathrm{~s}$ with a short break of $\sim 100 \mathrm{~s}$ between the blocks in which the subjects had to report the number of target stimuli.

Acquisition and analysis of MEG data. Magnetic fields were recorded in a magnetically shielded room using an MEG system with two separate dewars (Philips, Hamburg, Germany), each equipped with 31 gradiometer sensors of $70 \mathrm{~mm}$ baseline connected to two 32-channel Synamps amplifier systems (NeuroScan, El Paso, TX). The center gradiometer of the MEG dewar was placed above $\mathrm{C} 4$ and $\mathrm{C} 3$ of the international 10/20 system. Hence, both dewars were placed above the SII cortices. Involuntary movements were controlled using localization signals from 10 coils, which were attached at subjects' heads. This head localization was performed before and after each experimental block. The MEG signals were bandpassed between 0.3 and $300 \mathrm{~Hz}$ and digitized with a sampling rate of $1000 \mathrm{~Hz}$. Artifact removal was done according to visual inspection of all segments for the presence of ocular or head movements, which, on average, occurred in $<10 \%$ of trials. MEG data processing was performed with MATLAB (MathWorks, Natick, MA) using the "FieldTrip" open source toolbox (http://www.ru.nl/fcdonders/fieldtrip). Data were epoched relative to the onset of the electrical intracutaneous stimulus in a time window from -200 to $800 \mathrm{~ms}$ and sorted according to the conditions finger (middle, index), intensity (high, low), and task (target, nontarget). Furthermore, the nontarget trials were reduced by a randomization procedure to obtain the same number of trials as for the targets.

Spectral analysis. Spectral analyses of the MEG data were performed using sliding-window Fourier transformation and "multitaper" spectral estimates (Mitra and Pesaran, 1999). For lower frequencies up to $50 \mathrm{~Hz}$, a sliding-window Fourier analysis, using a Hanning window of $200 \mathrm{~ms}$, was used. For frequencies $>50 \mathrm{~Hz}$, the data were multiplied by $\mathrm{n}>1$ orthogonal tapers and Fourier transformed, and the $\mathrm{N}$ spectral estimates were finally averaged. In case of power estimation, the spectra for each individual taper were magnitude squared after Fourier transformation. As data taper, we use the leading $2 \mathrm{TW}-1$ prolate spheroidal (slepian) sequences, in which $\mathrm{T}$ denotes the length of the tapers and $\mathrm{W}$ denotes the half-bandwidth. These tapers optimally concentrate the spectral energy of the signal over the desired half-bandwidth W (Mitra and Pesaran, 1999). To obtain phase-locked and nonphase-locked information, induced power and evoked power were calculated. For calculation of evoked power, the averaged evoked field was transformed into the frequency domain, thus containing phase-locked information only. For induced power, the evoked field was subtracted from every single trial to eliminate phase-locked components and retain only nonphase-locked signals (Tallon-Baudry and Bertrand, 1999). Subsequently, each single trial was transformed to the frequency domain. Averaging across trials was finally performed in the frequency domain. Thus, all spectral estimates for the induced power contained only signal components that were nonphase-locked to stimulus onset (Pfurtscheller and Lopes da Silva, 1999; Tallon-Baudry and Bertrand, 1999). Spectral amplitudes were computed as the square root of the spectral power estimate. To characterize the temporal profile of spectral responses, we performed a timefrequency transformation of the MEG data using a sliding-window multitaper analysis. A window of $200 \mathrm{~ms}$ length was shifted over the data with a window step size of $10 \mathrm{~ms}$. Spectral smoothing of $20 \mathrm{~Hz}$ was achieved by 
using seven slepian tapers. The baseline spectrum was estimated as the average spectrum of the time-frequency transform across the interval from $200 \mathrm{~ms}$ before up to stimulus onset. For all power spectral analyses on the sensor level, responses were averaged over those 31 sensors that were centered over the left and right SII cortices.

Source reconstruction. To optimally capture the effect of interest, a frequency range was chosen for the grand mean dataset in which the effect of experimental variables was maximal and statistically significant on the sensor level. Power values for this specific frequency band were calculated for each condition and sensor. Then a difference-power time course was calculated. The latency for the source reconstruction was chosen from the time frequency representation (TFR) plot and the corresponding component. Subsequently, these difference-topology maps of the power spectra between the conditions were used for the source analysis. Source reconstruction of these time-frequency difference maps was performed on the basis of the brain morphology as obtained from a standard magnetic resonance image (MRI) [MNI (Montreal Neurological Institute) standard brain]. For the reconstruction of the neuronal sources for those spectral-difference maps, a linear three-dimensional cortical current density analysis (CCD) method implemented in the Curry software package (NeuroScan, Hamburg, Germany) was used (Ilmoniemi, 1991; Wagner, 1998). The CCD maps obtained in this way show the current flow distribution on the cortex, which can account for the potentials measured on the head surface. The ambiguity in the CCD model was removed by the "minimum norm constraint." This constraint uses a model term that is proportional to the square of the strength of the reconstructed currents. The regularization parameter was determined according to the number and location of cortical sources, whereby all sources were constrained to a surface representing the cortical gray matter. For the standard MRI, the segmented cortex with all gyri and sulci at $\sim 50,000$ sampled locations was used, hence the computation was restricted to the cortex. The orientation of the dipoles was not restricted. To account for the shapes of liquor, skull, and scalp, a realistic threecompartment boundary element method model was used as the volume conductor head model. Only sources with at least $75 \%$ of the strength at the maximum current density itself were considered. Under these circumstances (sensor distribution, source model used, and color scale), the drop from 100 to $75 \%$ happens within a volume of $3 \times 3 \times 3 \mathrm{~cm}$ (Fuchs et al., 1999).

Synchronization. To examine the phase relationship between the measured oscillations, the imaginary coherence (IMC) was calculated between pairs of sensors (Nolte et al., 2004). IMC is a useful tool to investigate true brain interactions, while suppressing effects that potentially arise from volume conduction. Whereas the latter occurs with precisely zero time lag, the imaginary part of coherency is only sensitive to interactions of two processes that occur with slight time lag relative to each other (Nolte et al., 2004). For IMC, only the imaginary part of the crosspectral-density matrix is calculated. Statistical significance of IMC was tested, on the one hand, by comparing coherence during the response with that occurring in the baseline for the same frequency range. Moreover, IMC, during the oscillatory response, was directly compared between the task conditions (target vs nontarget).

Statistical analysis. For statistical analysis, SPSS 10.0 (SPSS, Chicago, IL) was used. All power-change and IMC parameters were first checked with a one-sample Kolmogorov-Smirnov test for normal distribution. MEG results were then analyzed using a four-way factorial repeatedmeasures ANOVA testing effects of task condition (rare vs frequent), intensity (high vs low), side (ipsilateral vs contralateral), and finger (middle vs index) across subjects. Significant main and interaction effects were followed by post hoc paired $t$ tests. To identify significant changes of the grand mean time-frequency responses, a cluster-randomization approach was chosen (Maris et al., 2007), which controls the type-I error rate with respect to multiple comparisons. After averaging of the TFR data over subjects, $t$ statistics were computed for differences between baseline and poststimulus interval. A cluster-finding algorithm identified regions of contiguous significant power changes having a threshold of $p<0.05$. Subsequently, a null distribution was computed by randomly reassigning the data across subjects, time, and frequency and subsequently calculating the test statistics for the new set of clusters. A refer- ence distribution of cluster-level $t$ statistics was created from 1000 random draws. The $p$ value was then estimated according to the proportion of the randomization null distribution exceeding the observed cluster level test statistic. Finally, the maximum value of a time-frequency window was chosen from the significant cluster and fed into the ANOVA. Accounting for the time and frequency smoothing of the time-frequency decomposition, the windows were chosen to have a length of $200 \mathrm{~ms}$ and a bandwidth of $20 \mathrm{~Hz}$. On the single-subject level, the maximal value from each subject was allowed to vary in time and frequency within the previously defined grand average time-frequency windows.

\section{Results}

\section{Behavioral results}

Stimulus intensities for the low (I1) painful sensation were $0.22 \pm 0.07 \mathrm{~mA}$ for the index finger and $0.21 \pm 0.05 \mathrm{~mA}$ for the middle finger. The high stimulus intensities (I2) were $0.27 \pm 0.08$ $\mathrm{mA}$ for the index finger and $0.26 \pm 0.06 \mathrm{~mA}$ for the middle finger.

After each block, the subjects reported the total number of detected target stimuli. For all blocks, and all subjects, the mean percentage of detected targets was $95.5 \%$ with an SD of $6.1 \%$. Repeated-measures ANOVA for the task performance over blocks showed no significant block effect $\left(F_{(1,7)}=1.2 ; p=0.35\right)$. Thus, the accuracy of target detection over all blocks was high and stable.

\section{Pain induced and evoked high-frequency responses over somatosensory cortices}

Time-frequency analysis revealed four clearly distinct patterns of induced and one pattern of evoked oscillation in response to painful stimuli in both hemispheres (Fig. 2). In the time range from 50 to $250 \mathrm{~ms}$, a high $\gamma$ increase in the $60-80 \mathrm{~Hz}$ band could be observed, which will be labeled "pattern I," followed by a $\beta$ suppression between 150 and $350 \mathrm{~ms}$ in the $20 \mathrm{~Hz}$ band ("pattern II"). From 400 to $600 \mathrm{~ms}$, a power increase occurred in the $120-$ $140 \mathrm{~Hz} \gamma$-band ("pattern III"), followed by a $\beta$ rebound ("pattern IV") from 500 to $700 \mathrm{~ms}$. A significant evoked response, reflecting oscillatory activity phase-locked to the stimulus, occurred only in the $\delta$-frequency range $0-200 \mathrm{~ms}$ after stimulus onset ("pattern V"). Hence, pattern V reflects the time-frequency decomposition of the evoked field. Patterns II and IV look similar to the well known suppression and rebound of S1 activity. These five response patterns were most pronounced over the contralateral hemisphere but occurred with lower amplitude also over the hemisphere ipsilateral to the stimulated hand.

\section{Responses are modulated by task relevance and stimulus intensity}

To further analyze the modulation of induced and evoked oscillatory responses by attention (rare target vs frequent nontarget stimuli) and stimulus intensity (high vs low), we computed response spectra for each side, finger, task, and intensity individually. The maximal values of each subject for the different patterns were positively tested for normal distribution and then fed into the ANOVA. Results of the four-way repeated-measures ANOVA are listed in Table 1, and main effects with interactions are displayed in Figure 3. The high $\gamma$ pattern I showed a main effect for the recording side with stronger amplitude on the contralateral sensor side compared with the ipsilateral side $\left(F_{(1,19)}=7.3 ; p<\right.$ $0.05)$. Furthermore, a significant task by intensity interaction, because of a stronger power increase for the target condition during high-intensity stimuli $\left(F_{(1,19)}=11.0 ; p<0.01\right)$, could be found for $\gamma$ pattern I. The $\beta$ suppression pattern II showed a main effect for the recording side with stronger amplitude on the contralateral sensor side compared with the ipsilateral side $\left(F_{(1,19)}=\right.$ 
ROI ipsilateral

\section{induced power}

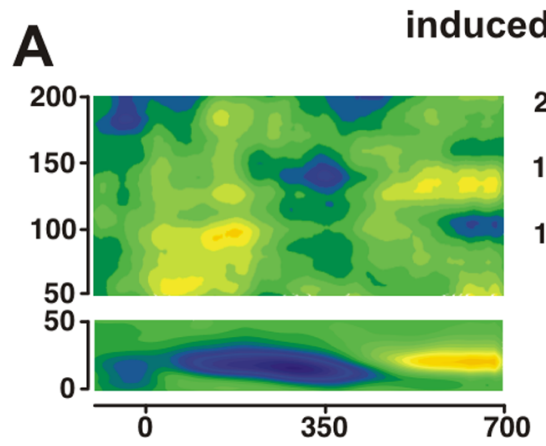

ROI contralateral

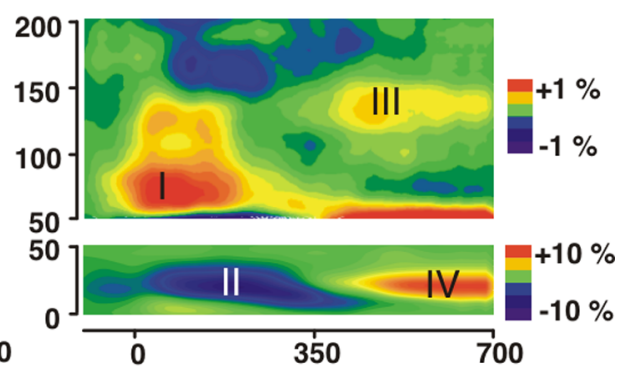

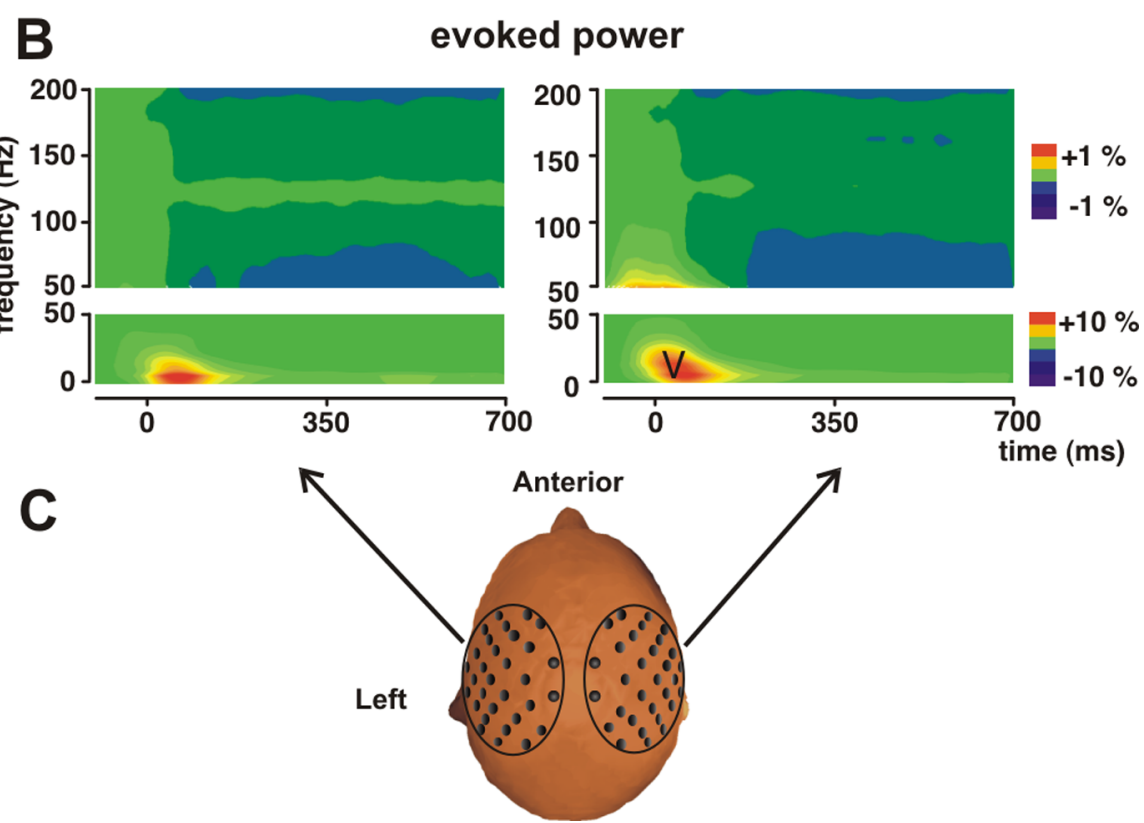

Figure 2. Pain stimulation yields induced and evoked oscillatory responses. $\boldsymbol{A}$, Grand mean time-frequency representation of induced oscillatory responses with high-intensity stimuli regardless of the task, averaged across 31 sensors for each side and 20 subjects. Responses are plotted as percentage signal change relative to the prestimulus baseline ( -200 to $0 \mathrm{~ms})$. In four distinct time-frequency regions (labeled I-IV), induced power changes can be observed. B, Calculation of evoked responses revealed only one significant peak (labeled V). C, Each dewar, which contains 31 sensors, builds one region of interest (ROI). In all subjects, stimuli were applied to the left hand.

$14.3 ; p<0.05)$. For $\gamma$ pattern III, no significant main effect of the recording side was observed, but a significant main effect of task $\left(F_{(1,19)}=6.3 ; p<0.05\right)$ could be found because of stronger oscillations for rare target compared with frequent nontarget stimuli. Furthermore, a significant task-by-intensity interaction because of stronger task effects for high-intensity compared with low-intensity stimuli could be found $\left(F_{(1,19)}=7.3 ; p<0.05\right)$. The $\beta$ rebound pattern IV had a main effect for the recording side with stronger amplitude on the contralateral sensor side compared with the ipsilateral side $\left(F_{(1,19)}=9.8 ; p<0.05\right)$. The evoked $\delta$ power pattern $\mathrm{V}$ showed main effects for intensity $\left(F_{(1,19)}=\right.$ $20.1 ; p<0.01)$ with more power increase for higher stimuli and for task $\left(F_{(1,19)}=19.4 ; p<0.01\right)$ because of stronger oscillations for rare target compared with frequent nontarget stimuli. In addition, a main effect for the recording side with stronger amplitude on the contralateral than on the ipsilateral sensor side $\left(F_{(1,19)}\right.$ $=6.1 ; p<0.05)$ was present for evoked $\delta$ pattern V. Furthermore, a significant task-by-intensity interaction because of stronger task effects for high-intensity compared with low-intensity stimuli could be found $\left(F_{(1,19)}=5.4 ; p<0.05\right)$. No main effect or any interactions could be found for the different stimulated fingers indicating that the site of stimulation had no influence on the results.

In summary, both early and late induced $\gamma$ oscillations were enhanced by task relevance of the stimuli, and even more so when the intensity of the stimuli was higher. Phase-locked early oscillations in the $\delta$-band $(\sim 3 \mathrm{~Hz})$ were modulated by stimulus intensity and task relevance and also showed sensitivity to an interaction of both factors. Interestingly, the remaining response patterns II and IV, which reflect the $\beta$ suppression and rebound, albeit clearly reflecting stimulus-related processing, were not modulated by either of these factors or their interactions.

\section{Source localization of $\delta$ - and $\gamma$-band responses}

Source localization was performed to estimate the spatial distribution for the oscillatory responses that could be identified as statistically significant in the grand mean time-frequency analyses. Therefore, difference datasets calculated by subtracting the corresponding time-frequency data from each other were used for the current density estimation. Such difference datasets were calculated between frequent nontarget and rare target stimuli for pattern III (120-140 Hz, 400-600 ms) and pattern V (3 Hz, 0-200 ms). Furthermore, a difference dataset was calculated for pattern $\mathrm{V}$ between high and low stimuli.

The activation difference for pattern III, which reflects the task relevance effect on the late induced $\gamma$ response, was localized bilaterally over sensory motor sides (Fig. 4A) with Talairach coordinates $x=$ $-54.3, y=-18.5$, and $z=24.2$ for the ipsilateral side and $x=51.9, y=-24.3$, and $z=30.4$ for the contralateral side, respectively. The spatial correlate of the task relevance effect of pattern $\mathrm{V}$ was found over the contralateral sensorimotor cortex (Fig. 4B) with Talairach coordinates $x=51.8, y=-1.1$, and $z=46.9$. Additionally, the anatomical correlate of the stimulus intensity effect for pattern $\mathrm{V}$ was also found at contralateral sensorimotor sites (Fig. 4C) with the coordinates $x=42.2, y=6.8$, and $z=51.5$.

\section{Synchronization between both sensorimotor cortices}

Synchronization between the sensorimotor cortices was only analyzed for the activation pattern III $(120-140 \mathrm{~Hz}, 400-600 \mathrm{~ms})$, because this pattern showed a significant modulation by task relevance, but the activation was independent of the sensor side. IMC was calculated for pattern III and the baseline epochs in the same frequency range before stimulus onset for both the nontarget and target conditions. Statistically, significant increases in IMC across subjects, both with respect to the number of coherent pairs of sensory and the strength of phase coupling, were identified using right-sided $t$ test statistics. Relative to baseline, an increase in both the number of coherent pairs and the strength of 
Table 1. Statistical results with main effects for the different oscillation patterns (I-V) after application of the ANOVA

\begin{tabular}{|c|c|c|c|c|c|c|c|c|c|c|}
\hline & \multicolumn{2}{|c|}{$\mathrm{l}: 60-80 \mathrm{~Hz}, 50-250 \mathrm{~ms}$} & \multicolumn{2}{|c|}{$\mathrm{Il}: 20 \mathrm{~Hz}, 150-350 \mathrm{~ms}$} & \multicolumn{2}{|c|}{ III: $120-140 \mathrm{~Hz}, 400-600 \mathrm{~ms}$} & \multicolumn{2}{|c|}{ IV: $20 \mathrm{~Hz}, 500-700 \mathrm{~ms}$} & \multicolumn{2}{|c|}{$\mathrm{V}: 3 \mathrm{~Hz}, 0-200 \mathrm{~ms}$} \\
\hline & $p$ value & Fvalue & $p$ value & Fvalue & $p$ value & Fvalue & $p$ value & Fvalue & $p$ value & Fvalue \\
\hline Task & n.s. & n.s. & n.s. & n.s. & $<0.05$ & 6.3 & n.s. & n.s. & $<0.01$ & 19.4 \\
\hline Intensity & n.s. & n.s. & n.s. & n.s. & n.s. & n.s. & n.s. & n.s. & $<0.01$ & 20.1 \\
\hline Finger & n.s. & n.s. & n.s. & n.s. & n.s. & n.s. & n.s. & n.s. & n.s. & n.s. \\
\hline Side & $<0.05$ & 7.3 & $<0.05$ & 14.3 & n.s. & n.s. & $<0.05$ & 9.8 & $<0.05$ & 6.1 \\
\hline Task $\times$ intensity & $<0.01$ & 11 & n.s. & n.s. & $<0.05$ & 7.3 & n.s. & n.s. & $<0.05$ & 5.4 \\
\hline
\end{tabular}

n.s., Not significant.

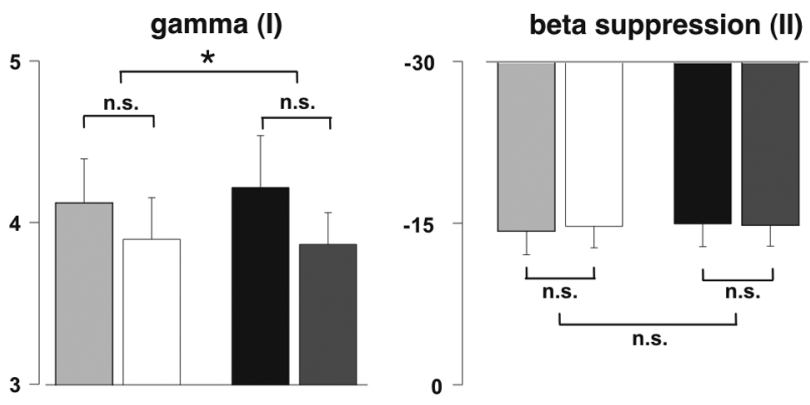

gamma (III)
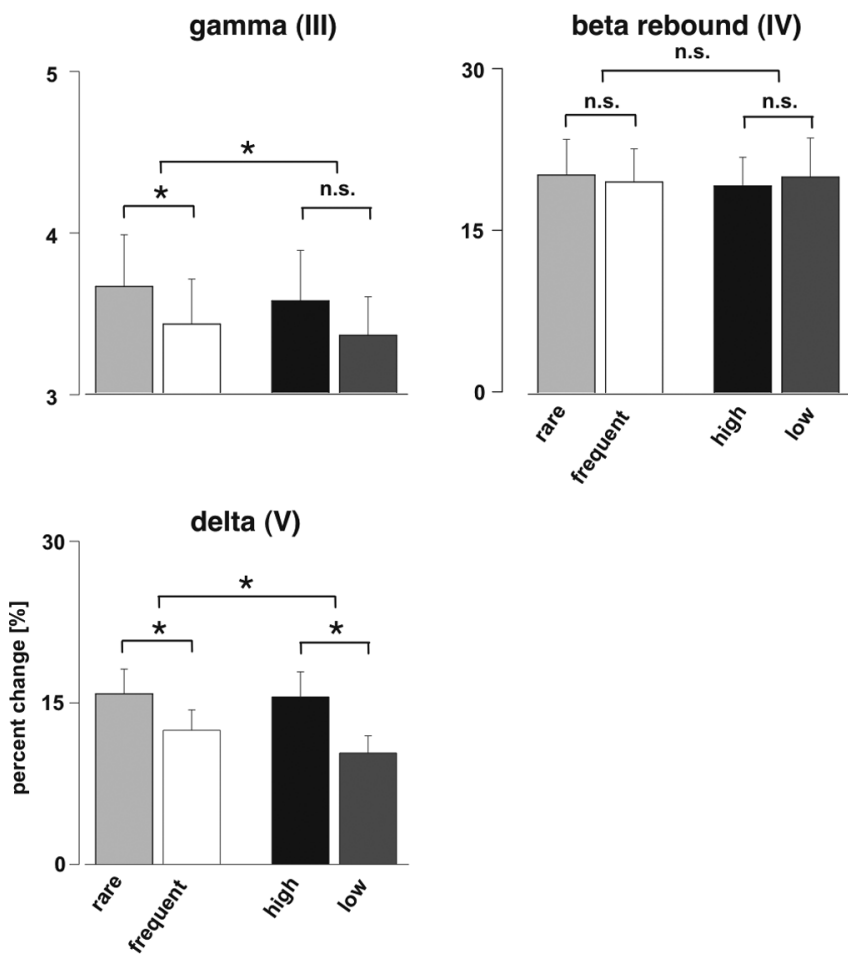

Figure 3. Statistical results for the five different time-frequency activation patterns $[\gamma(\mathrm{l})$ : $60-80 \mathrm{~Hz}, 50-250 \mathrm{~ms} ; \beta$ suppression (II): $20 \mathrm{~Hz}, 150-350 \mathrm{~ms} ; \gamma$ (III): $120-140 \mathrm{~Hz}, 400-600$ ms; $\beta$ rebound (IV): $20 \mathrm{~Hz}, 500-700 \mathrm{~ms} ; \delta(\mathrm{V}): 3 \mathrm{~Hz}, 0-200 \mathrm{~ms}$. Error bars indicate SE. $\gamma$ response I had a significant task-by-intensity interaction because of a stronger power increase for the target condition during high-intensity stimuli. The $\beta$ suppression II and rebound IV had no significant effects for stimulus intensity or task relevance. High $\gamma$ response III had significant stronger oscillations for rare target compared with frequent nontarget stimuli. Furthermore, a significant task-by-intensity interaction because of stronger task effects for high-intensity compared with low-intensity stimuli could be found for this high $\gamma$ response. The evoked $\delta$ power pattern $\mathrm{V}$ had a power increase for high intensity and rare target stimuli with a significant task-by-intensity interaction because of a stronger task effects for high-intensity compared with low-intensity stimuli. n.s., Nonsignificant. ${ }^{*} p<0.05$.
IMC was observed for response pattern III in the target as well as the nontarget condition (Fig. 5). Significance was also tested by direct comparison of both conditions, showing that the increase in interhemispheric connectivity was stronger for the target condition compared with the nontarget stimuli (Fig. 5).

\section{Discussion}

The results of the present study demonstrate that several brain processes underlying pain perception can be subject to attentional modulation. For the first time, to our knowledge, we describe convergent effects of focused attention and pain intensity on high-frequency oscillations and their interhemispherical degree of synchronization. For this, we systematically compared phase-locked (i.e., evoked) and nonphase-locked (i.e., induced) activities by using an oddball paradigm with painful intracutaneous electrical stimuli in healthy subjects. After painful stimulation, four different induced time-frequency patterns and one evoked oscillatory pattern emerged. The well known $\beta$ suppression with $\beta$ rebound could be observed. For the high $\gamma$-band, two power increases were observed: one between 50 and $250 \mathrm{~ms}$ (60-80 Hz; pattern I) and the other between 400 and $600 \mathrm{~ms}$ (120-140 Hz; pattern III). Analysis of evoked responses revealed an early $\delta$ increase $(3 \mathrm{~Hz}, 0-200 \mathrm{~ms}$; pattern $\mathrm{V})$, which mainly reflects the evoked field. Only patterns III and V exhibited consistent dependency on attention, as revealed by the contrast between rare target and frequent nontarget stimuli, and on pain intensity. Source localization for these patterns revealed activation at contralateral sensorimotor regions for evoked $\delta$ power, but bilateral and slightly more ventral sensorimotor activation for the late induced $\gamma$-band response. These results suggest a complex interplay between different sensorimotor regions involving oscillatory processes in different frequency bands during early and late phases of nociceptive information processing, which can be modulated by top-down influences.

The topography of our findings is consistent with that of other studies, which examined the classical evoked responses at lowfrequency bandwidths. In particular, MEG studies have demonstrated a bilateral SII and a simultaneous contralateral SI activation after painful laser stimulation (Ploner et al., 1999, 2002). Localization of pattern $\mathrm{V}$, which corresponds to the timefrequency transform of the evoked field, confirms these findings, being most prominent contralateral to the stimulation site. However, the variability of the coordinates for both sides are within the range of spatial resolution typically assumed for source reconstruction methods. Our finding of a task-related effect for this early phase-locked response suggests that attentional modulation can affect pain processing already at the level of the sensorimotor cortex. Going beyond previous studies, our results provide evidence for enhanced $\gamma$-band activity over both sensorimotor cortices and, in particular, for enhanced synchronization between sensorimotor regions of both hemispheres during focused attention to painful stimuli. The enhanced functional coupling be- 

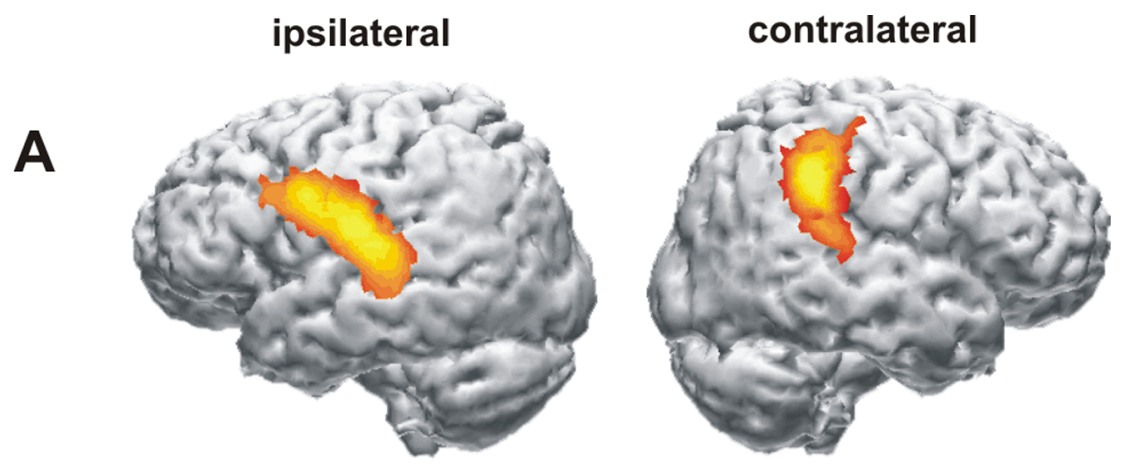

B
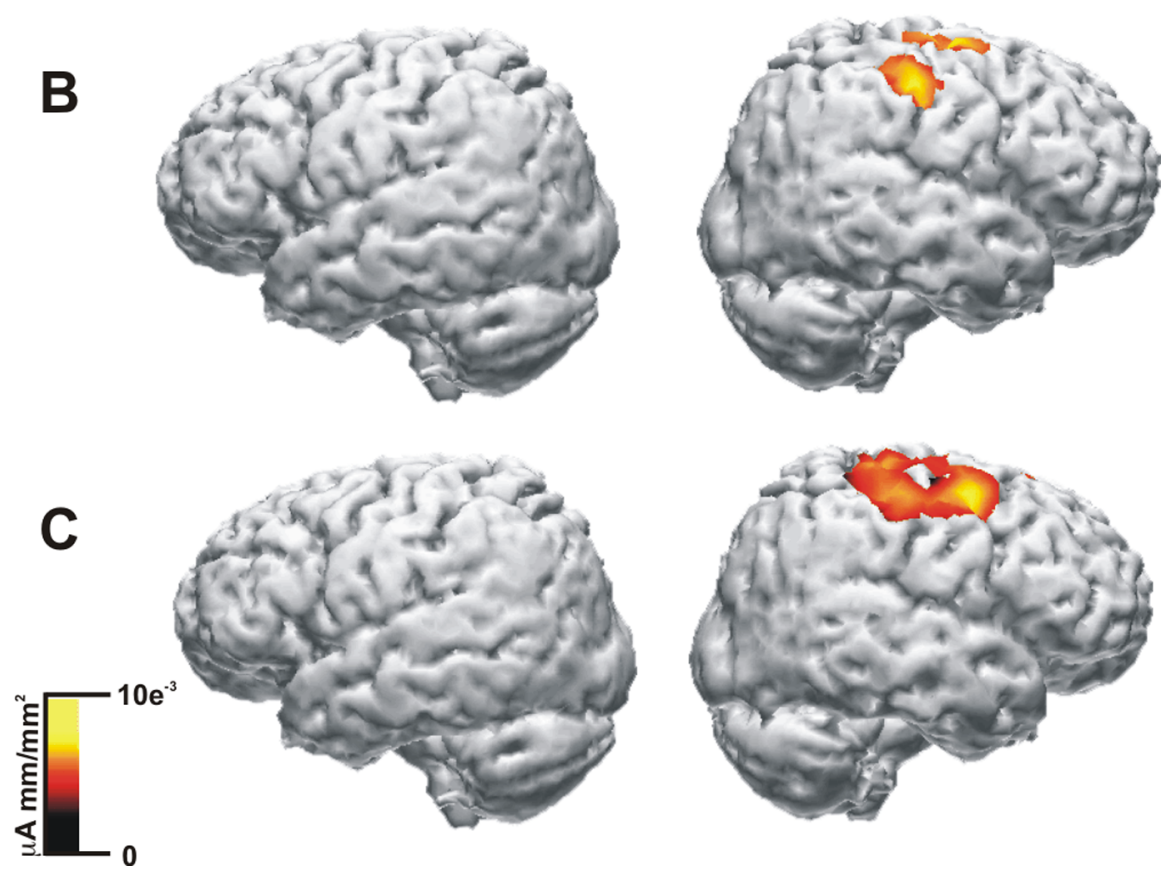

Figure 4. Grand mean source reconstruction results. $\boldsymbol{A}$, For $\gamma$ pattern III (120-140 Hz, $400-600 \mathrm{~ms})$, which showed the attention effect, activations were found bilaterally in regions covering the sensorimotor cortex. $\boldsymbol{B}$, For the early evoked $\delta$ pattern $\mathrm{V}(3 \mathrm{~Hz}, 0-200 \mathrm{~ms})$, the correlate of the attention effect was observed in regions more dorsal and only at the contralateral sensorimotor cortex. C, For the same pattern V, which showed significant effects for the stimulus intensity, activation was also found at the contralateral sensorimotor cortex.
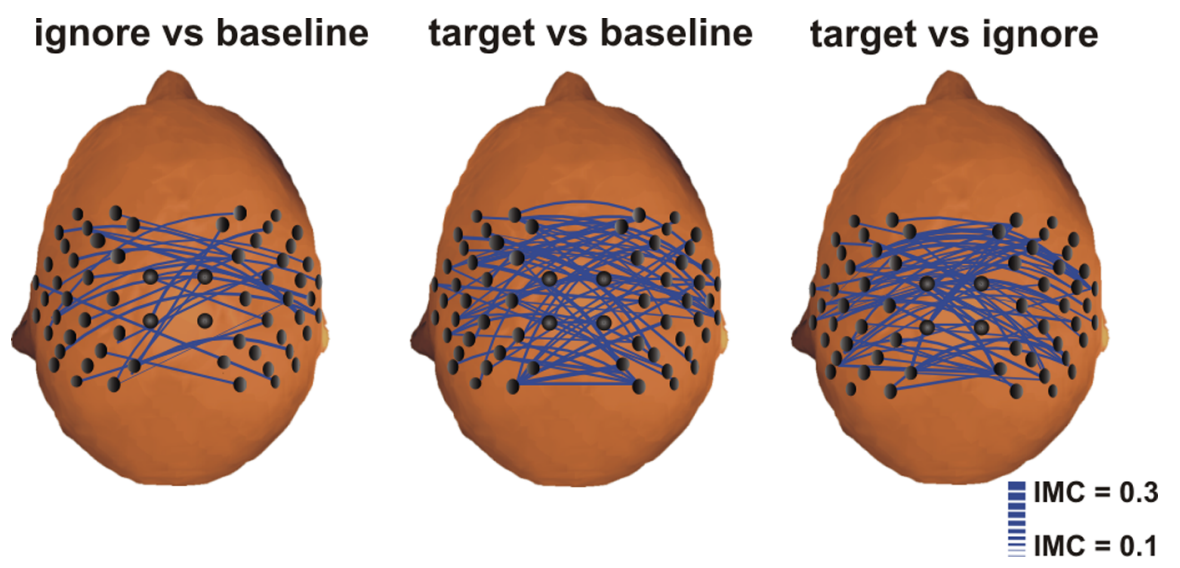

Figure 5. Attention-dependent modulation of the IMC across hemispheres (below). Only interhemispheric combinations are displayed, and the thickness of the lines reflects the strength of the IMC. An increase in synchronization was observed for activation pattern III (120-140 Hz, $400-600 \mathrm{~ms})$ for all subjects $(n=20)$. In the left panel, a significant increase in IMC was calculated between pattern III and the corresponding baseline before stimulus onset for the nontarget condition. In the middle panel, the same is shown for the target condition. Direct comparisons between target and nontarget conditions were calculated in the right panel. tween both hemispheres, which receive direct input from the thalamus (Ploner et al., 1999), could be important for optimally integrating the behavioral relevance of somatosensory signals regarding momentary task demands and bodily threat. Bilateral SII activation has been suggested to play a role for integration of sensory information from the two body halves and might be important for the maintenance of the body schema (Lin and Forss, 2002).

Oscillatory processes, particularly those in the $\gamma$-frequency range, have been shown to be modulated by both bottom-up (i.e., stimulus-driven) and top-down (i.e., subject-driven) factors (Engel et al., 2001). Animal and human experiments have demonstrated that coherent activity in the $\gamma$-band is enhanced during attentional selection of sensory information (for review, see Herrmann et al., 2004). Strong attentional effects on $\gamma$-band responses have been observed in visual areas such as V4 in awake behaving monkeys when the attention was shifted toward the stimulus processed by the recorded cells (Fries et al., 2001; Taylor et al., 2005). The same $\gamma$ enhancement during attention was found in human experiments using EEG and MEG for the visual system (Gruber et al., 1999), auditory processing (Tiitinen et al., 1993; Debener et al., 2003), and recently also for somatosensory processing (Bauer et al., 2006). Single-neuron recordings in awake behaving monkeys have shown that cells in SII that respond to tactile stimuli are also sensitive to attention and behavioral context (Steinmetz et al., 2000). Synchrony in SII markedly increased when the monkey attentively processed the somatosensory information. Moreover, changes in synchrony were stronger when the somatosensory discrimination task was more difficult.

Notably, these findings could be extended to pain processing in a recent study conducted in neurosurgical awake patients by demonstrating attention to painful laser stimuli to enhance intracranially recorded synchrony between distinct cortical pain-related regions (Ohara et al., 2006). The pattern of synchrony changed between the primary somatosensory cortex, the medial frontal cortex, and the insula as the patient switched from a distracted state to attention to the stimuli by counting the total number. In our study, attention to pain stimuli did not change over blocks and time but was directed to one finger, which was stimulated in alternating order in each block. Global attention was present during the entire experi- 
ment, but the focus was to count the target stimuli. Therefore, enhanced $\gamma$ power and synchronization are unlikely to be a result of higher arousal or global attentional state of the pain system but seem to correlate specifically with the shift to the attended body location, especially when stronger painful stimuli are applied.

The findings of enhanced $\gamma$-band activity and synchronization in our study are compatible with the suggestion that attention might exert its effect by modulating the coherence of ongoing oscillations selectively for the subset of neurons involved in encoding the target stimuli. On presentation of the appropriate stimulus, neurons exhibiting such coherent subthreshold oscillations might show well synchronized responses that are transmitted more reliably than nonsynchronized responses. In this manner, synchrony could help to selectively route the attended signals toward downstream areas involved in stimulus evaluation and action planning (Salinas and Sejnowski, 2001; Engel et al., 2001; Fries, 2005; Womelsdorf et al., 2007). In case of pain stimuli, synchrony may bias the routing of signals toward the anterior cingulate or the dorsolateral prefrontal cortex, which are regarded important for emotional evaluation, monitoring, and descending control of pain (Lorenz et al., 2003). Because the task in our study did not allow the evaluation of pain ratings after each stimulus, our results do not provide a direct link between perceived painfulness and brain oscillations. However, a recent study by Gross et al. (2007) has demonstrated that the strength of pain-induced $\gamma$ oscillations in SI is related to the subjective perception of pain, suggesting that enhanced neural coherence may indeed facilitate the preferential routing of important information in nociceptive processing.

Attention is a major determinant of perception, which can profoundly change behavior and pain experience (Eccleston and Crombez, 1999). Clinical evidence suggests that attentional mechanisms may be involved in the pathogenesis of some chronic clinical pain stages (Vlaeyen and Linton, 2000). Accordingly, clinical interventions using modulation of attention (distraction) have been demonstrated to relieve pain in chronic pain patients (McCracken and Turk, 2002). These findings were confirmed by several experimental studies, although experimental models presumably only partly account for the complex nature of chronic pain. Whereas patients with chronic pain problems seem to selectively attend to sensory aspects of pain, the degree by which pain distracts attention from concurrent tasks appears to depend on the evaluation of pain stimuli as threatening or worrying (Eccleston and Crombez, 1999). Patients that are highly fearful of pain may not only selectively attend to pain-related information but have difficulty disengaging from those stimuli (Dehghani et al., 2003). Furthermore, the processing capacity in chronic pain patients for cognitive and mental effort seems to be diminished (Veldhuijzen et al., 2006). One possible neuronal correlate for this complex deficit in central pain modulation could be an "oversynchronization" among pain-related cortical areas, leading to an uncontrolled spread of signals even in the case of weak or absent nociceptive inputs. Thus, the dynamic control of neuronal signal flow might be disturbed, preventing an appropriate context-dependent modulation of the gain of neural signals and reducing the capacity for descending control of nociceptive afferent inputs. As assumed for states of chronic pain, such an oversynchronization might be viewed as the result of a learning process with resulting central neuroplastic changes (Flor and Diers, 2007). However, this speculation needs to be proven by investigation of pain-induced oscillatory activity and, in particular, of $\gamma$-band coherence in chronic pain patients.

In conclusion, we found that directed attention to pain was associated with an increase in induced oscillations and synchrony in the high $\gamma$-band over both hemispheres. We suggest that augmentation of these oscillatory activations may be one mechanism by which attention facilitates processing of neural responses, leading to enhanced saliency of sensory signals and preferential routing of the respective information through the cortical network. We suggest that analyzing oscillations and their interactions may be a promising approach to investigate the mechanisms of cortical pain processing.

\section{References}

Aldrich S, Eccleston C, Crombez G (2000) Worrying about chronic pain: vigilance to threat and misdirected problem solving. Behav Res Ther 38:457-470.

Bauer M, Oostenveld R, Peeters M, Fries P (2006) Tactile spatial attention enhances gamma-band activity in somatosensory cortex and reduces lowfrequency activity in parieto-occipital areas. J Neurosci 26:490-501.

Becker DE, Haley DW, Urena VM, Yingling CD (2000) Pain measurement with evoked potentials: combination of subjective ratings, randomized intensities, and long interstimulus intervals produces a P300-like confound. Pain 84:37-47.

Bromm B, Lorenz J (1998) Neurophysiological evaluation of pain. Electroencephalogr Clin Neurophysiol 107:227-253.

Bromm B, Meier W (1984) The intracutaneous stimulus: a new pain model for algesimetric studies. Methods Find Exp Clin Pharmacol 6:405-410.

Chen AC, Herrmann CS (2001) Perception of pain coincides with the spatial expansion of electroencephalographic dynamics in human subjects. Neurosci Lett 297:183-186.

Croft RJ, Williams JD, Haenschel C, Gruzelier JH (2002) Pain perception, hypnosis and $40 \mathrm{~Hz}$ oscillations. Int J Psychophysiol 46:101-108.

Debener S, Herrmann CS, Kranczioch C, Gembris D, Engel AK (2003) Topdown attentional processing enhances auditory evoked gamma band activity. Neuroreport 14:683-686.

Dehghani M, Sharpe L, Nicholas MK (2003) Selective attention to painrelated information in chronic musculoskeletal pain patients. Pain 105:37-46.

Dowman R (2004) The pain-evoked P2 is not a P3a event-related potential. Brain Topogr 17:3-12.

Eccleston C, Crombez G (1999) Pain demands attention: a cognitiveaffective model of the interruptive function of pain. Psychol Bull 125:356-366.

Eccleston C, Crombez G, Aldrich S, Stannard C (2001) Worry and chronic pain patients: a description and analysis of individual differences. Eur J Pain 5:309-318.

Engel AK, Singer W (2001) Temporal binding and the neural correlates of sensory awareness. Trends Cogn Sci 5:16-25.

Engel AK, Fries P, Singer W (2001) Dynamic predictions: oscillations and synchrony in top-down processing. Nat Rev Neurosci 2:704-716.

Flor H, Diers M (2007) Limitations of pharmacotherapy: behavioral approaches to chronic pain. Handb Exp Pharmacol 177:415-427.

Fries P (2005) A mechanism for cognitive dynamics: neuronal communication through neuronal coherence. Trends Cogn Sci 9:474-480.

Fries P, Reynolds JH, Rorie AE, Desimone R (2001) Modulation of oscillatory neuronal synchronization by selective visual attention. Science 291:1560-1563.

Fuchs M, Wagner M, Kohler T, Wischmann HA (1999) Linear and nonlinear current density reconstructions. J Clin Neurophysiol 16:267-295.

Gray CM, Singer W (1995) Visual feature integration and the temporal correlation hypothesis. Annu Rev Neurosci 18:555-586.

Gross J, Schnitzler A, Timmermann L, Ploner M (2007) gamma oscillations in human primary somatosensory cortex reflect pain perception. PLoS Biol 5:e133.

Gruber T, Muller MM, Keil A, Elbert T (1999) Selective visual-spatial attention alters induced gamma band responses in the human EEG. Clin Neurophysiol 110:2074-2085.

Herrmann CS, Munk MH, Engel AK (2004) Cognitive functions of gammaband activity: memory match and utilization. Trends Cogn Sci 8:347-355.

Ilmoniemi RJ (1991) Estimates of neuronal current distributions. Acta OtoLaryngol Suppl 491:80-87.

Legrain V, Guerit JM, Bruyer R, Plaghki L (2002) Attentional modulation of the nociceptive processing into the human brain: selective spatial atten- 
tion, probability of stimulus occurrence, and target detection effects on laser evoked potentials. Pain 99:21-39.

Lin YY, Forss N (2002) Functional characterization of human second somatosensory cortex by magnetoencephalography. Behav Brain Res 135:141-145.

Lorenz J, Garcia-Larrea L (2003) Contribution of attentional and cognitive factors to laser evoked brain potentials. Neurophysiol Clin 33:293-301.

Lorenz J, Minoshima S, Casey KL (2003) Keeping pain out of mind: the role of the dorsolateral prefrontal cortex in pain modulation. Brain 126:10791091.

Maris E, Schoffelen JM, Fries P (2007) Nonparametric statistical testing of coherence differences. J Neurosci Methods 163:161-175.

McCracken LM, Turk DC (2002) Behavioral and cognitive-behavioral treatment for chronic pain: outcome, predictors of outcome, and treatment process. Spine 27:2564-2573.

Mitra PP, Pesaran B (1999) Analysis of dynamic brain imaging data. Biophys J 76:691-708.

Nolte G, Bai O, Wheaton L, Mari Z, Vorbach S, Hallett M (2004) Identifying true brain interaction from EEG data using the imaginary part of coherency. Clin Neurophysiol 115:2292-2307.

Ohara S, Crone NE, Weiss N, Lenz FA (2006) Analysis of synchrony demonstrates "pain networks" defined by rapidly switching, task-specific, functional connectivity between pain-related cortical structures. Pain 123:244-252.

Peyron R, Laurent B, Garcia-Larrea L (2000) Functional imaging of brain responses to pain. A review and meta-analysis. Neurophysiol Clin 30:263-288.

Pfurtscheller G, Lopes da Silva FH (1999) Event-related EEG/MEG synchronization and desynchronization: basic principles. Clin Neurophysiol 110:1842-1857.

Ploner M, Schmitz F, Freund HJ, Schnitzler A (1999) Parallel activation of primary and secondary somatosensory cortices in human pain processing. J Neurophysiol 81:3100-3104.

Ploner M, Gross J, Timmermann L, Schnitzler A (2002) Cortical represen- tation of first and second pain sensation in humans. Proc Natl Acad Sci USA 99:12444-12448.

Salinas E, Sejnowski TJ (2001) Correlated neuronal activity and the flow of neural information. Nat Rev Neurosci 2:539-550.

Schnitzler A, Ploner M (2000) Neurophysiology and functional neuroanatomy of pain perception. J Clin Neurophysiol 17:592-603.

Siegel M, Donner TH, Oostenveld R, Fries P, Engel AK (2007) Highfrequency activity in human visual cortex is modulated by visual motion strength. Cerebral Cortex 17:732-741.

Singer W (1999) Neuronal synchrony: a versatile code for the definition of relations? Neuron 24:49-65, 111-125.

Steinmetz PN, Roy A, Fitzgerald PJ, Hsiao SS, Johnson KO, Niebur E (2000) Attention modulates synchronized neuronal firing in primate somatosensory cortex. Nature 404:187-190.

Tallon-Baudry C, Bertrand O (1999) Oscillatory gamma activity in humans and its role in object representation. Trends Cogn Sci 3:151-162.

Taylor K, Mandon S, Freiwald W. A, Kreiter AK (2005) Coherent oscillatory activity in monkey V4 predicts successful allocation of attention. Cerebral Cortex 15:1424-1437.

Tiitinen H, Sinkkonen J, Reinikainen K, Alho K, Lavikainen J, Naatanen R (1993) Selective attention enhances the auditory $40-\mathrm{Hz}$ transient response in humans. Nature 364:59-60.

Veldhuijzen DS, Kenemans JL, van Wijck AJ, Olivier B, Kalkman CJ, Volkerts ER (2006) Processing capacity in chronic pain patients: a visual eventrelated potentials study. Pain 121:60-68.

Vlaeyen JW, Linton SJ (2000) Fear-avoidance and its consequences in chronic musculoskeletal pain: a state of the art. Pain 85:317-332.

Wagner M (1998) Rekonstruktion neuronaler Ströme aus bioelektrischen und biomagnetischen Messungen auf der aus MR-Bildern segmentierten Hirnrinde. Aachen, Germany: Shaker Verlag.

Womelsdorf T, Schoffelen J-M, Oostenveld R, Singer W, Desimone R, Engel AK, Fries $P$ (2007) Modulation of neuronal interactions through neuronal synchronization. Science 316:1609-1612. 